\title{
SHIELDING EFFECTIVENESS OF PLANAR MATERIALS: (semi)-STANDARDIZED MEASUREMENTS FROM LF TO $\mu \mathrm{W}$
}

\author{
Johan Catrysse, Davy Pissoort, Filip Vanhee \\ ReMi, KULeuven Technology Campus Oostende, Zeedijk 10, B 8400 Oostende (Belgium \\ Email:Davy.Pissoort@kuleuven.be
}

\begin{abstract}
Due to the impact of higher and higher frequencies in a lot of applications, the Shielding Effectiveness (SE) characterisation of materials is expanding from LF up to frequencies well above $1 \mathrm{GHz}$. Additionally, requirements with respect to energy-efficiency necessitate the use of light-weight shielding materials such as composites, especially in aerospace applications. A typical concern about composite materials is their internal homogeneity, which may result in a nearly isotropic up to a full anisotropic material type. This paper gives an overview of (semi)-standardized measurement methodologies from Low Frequencies (LF) up to the $\mu$ Wave $(\mu W)$ region, including discussions on different $\mathrm{SE}$ values obtained by different methodologies.
\end{abstract}

\section{INTRODUCTION}

Due to the impact of higher and higher frequencies, the SE characterization of shielding materials and components will be needed over the broad frequency range from LF to $\mu$ Wave frequencies.

In a lot of mobile applications, but also in large structures such as in aerospace applications, weight becomes a very important parameter. Plastics can be painted or injected with conductive fibers, but in a lot of cases, composite materials can combine requirements on mechanical behavior and the need of some shielding capability. A typical issue about composite materials is their structure, behaving from a nearly isotropic into full anisotropic material type. In this paper, research results on the validity of some shielding measurements are summarized for three types of materials, ranging from isotropic into anisotropic structure.

This paper gives an overview of measurement methodologies from Low Frequencies (LF) up to the $\mu$ Wave $(\mu \mathrm{W})$ region. For each topic, standardized as well as semi-standardized methods will be discussed. Correct interpretations and possible pitfalls are discussed, especially when different methods lead to different $\mathrm{SE}$ values.

\section{LF MAGNETIC FIELD SE}

For the characterization of the LF magnetic shielding two setups are available. Both are based on the use of loop antennas, but the physical setup is different.
One is referenced in NSA 94-106 (formerly NSA 65-6) [3] and is known as the parallel setup, where both transmitting and receiving antennas are in parallel planes, and parallel with the plane of the sample under test. This is sketched in figure 1.

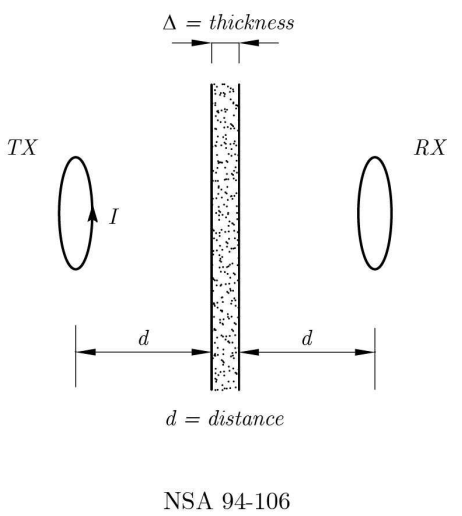

Figure 1. Setup for NSA 94-106 LF magnetic SE

The other one is referenced in IEEE Std 299 (formerly MIL STD 285-[2]) and is known as the coplanar setup, where both antennas are located in the same plane, but perpendicular to the sample. This is sketched in figure 2 .

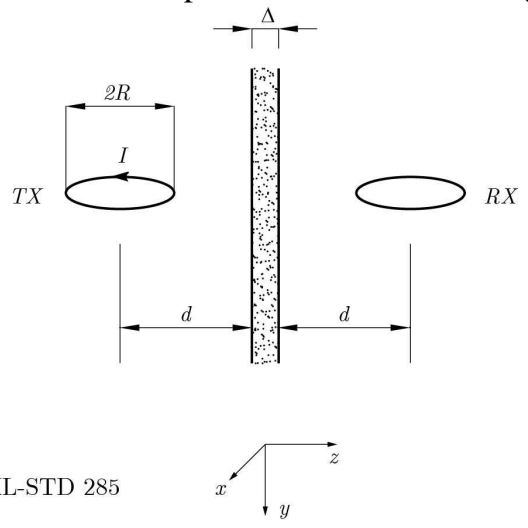

Figure 2. Setup for IEEE Std 299 LF magnetic SE

When performing SE measurements using both methodologies, but identical samples and equipment, different results are obtained for the resulting SE values.

In the NSA 94-106 configuration, tangential components of the magnetic field are only generated in points away from the loop axis. The larger the region that may be taken into account, the larger the observed effect in the field distribution. This results in a 
dependency of the measured $\mathrm{SE}$ on the radius $\mathrm{R}$ of the transmitting antenna.

In the IEEE Std. 299 configuration, tangential components are exactly those who propagate through the shield. The difference of the dimensions of the transmitting loop antenna is not so relevant when the radius $\mathrm{R}$ of the antenna's is changed.

Measurements are performed using a metal cage with an open window of minimum $50 \times 50 \mathrm{~cm}$ in one wall, requiring samples of at least $60 \times 60 \mathrm{~cm}$.

The measurement setup is sketched in figure 3 .

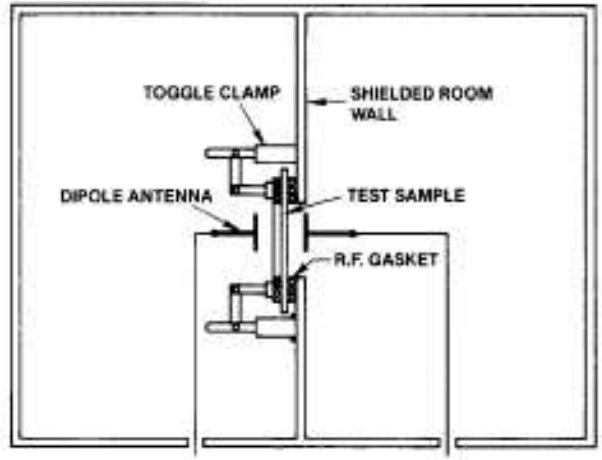

Figure 3. Basic principle of IEEE Std 299 like measuring setup for planar materials

A more detailed view of the fixture in order to clamp the samples is shown in the figures 4 .

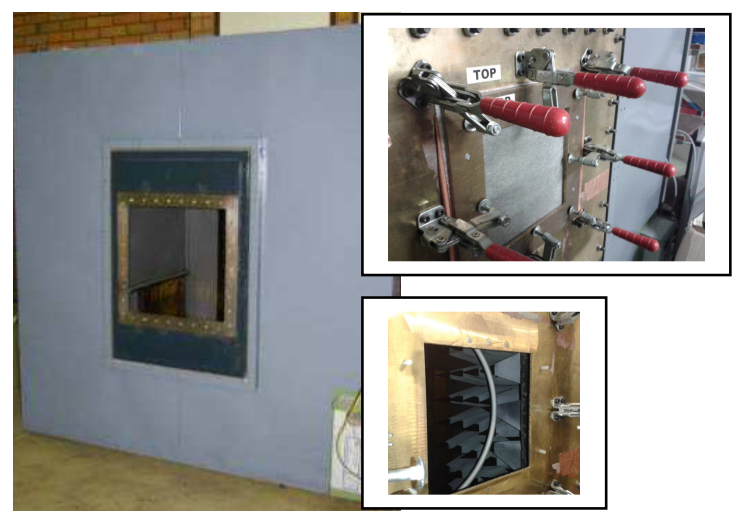

Figure 4. Picture of an IEEE Std 299 like "fixture"

The next figure shows the measured SE values between $20 \mathrm{KHz}$ and $20 \mathrm{MHz}$ for a thin aluminium foil. Theoretical difference of $10 \mathrm{~dB}$ between both measurement methodologies is observed, but also the extra effect of changing antenna's with another radius in the NSA 94-106 setup (needed to cover the whole frequency spectrum), and corrected assuming a continuous behaviour of the SE (red line in figure 5). A more theoretical analysis is given in reference [4].

The next graph is showing three curves:

- NSA 94-106, as measured (blue)

- NSA 94-106 corrected for changing antenna radius R (red)

- IEEE Std 299, as measured (black)



Figure 5. Magnetic field SE in the lower frequency range (20 KHz-20MHz) using IEEE 299 \& NSA 94-106

\section{SE UP TO 1 GHz FOR PLANE WAVE EM}

In the range of $10 \mathrm{MHz}$ up to $1 \mathrm{GHz}$, ASTM D4935 standard can be used for smaller samples $(15 \mathrm{~cm}$ diameter), but an IEEE 299 setup using dipoles, biconical and logper antenna's is also commonly used. Due to the circular cross section of the ASTM D4935 cell, a radial symmetry of the E-field is generated. No discrimination of direction-dependent properties of the material (anisotropy) is possible.

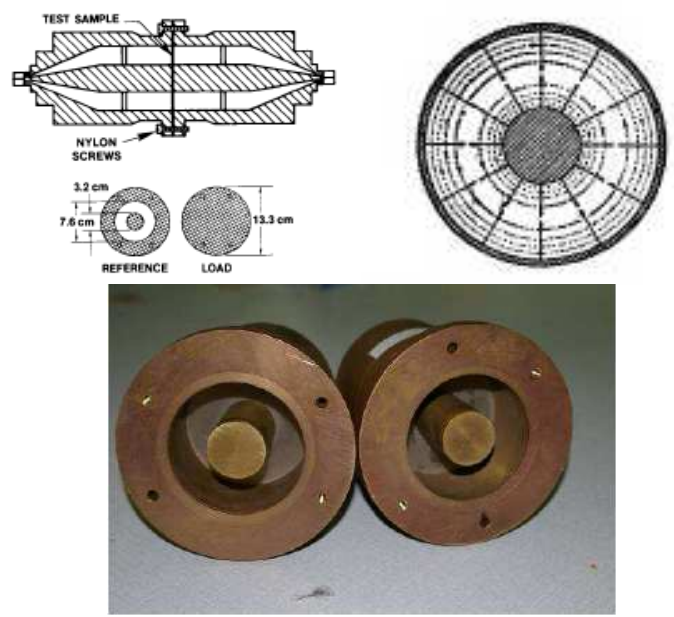

Figure 6. ASTM D 4935 cell and the field polarization

In the IEEE 299 setup, a linear polarization directly related to the positioning of the active dipoles of a logper antenna is obtained, and discrimination of anisotropy is possible.
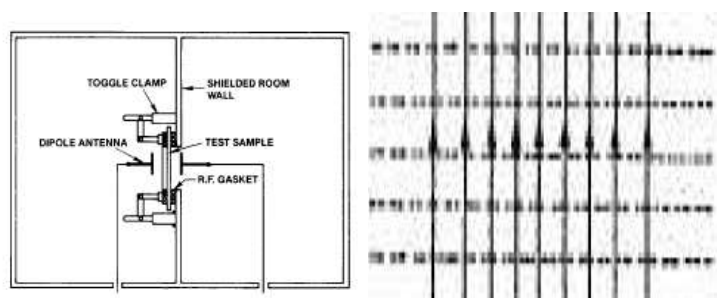

Figure 7. IEEE 299 setup and the E-field polarization 
The next examples show the results for three type of materials:

- full isotropic material

- semi isotropic material

- full anisotropic material

SE van sample 2 - astm D4935
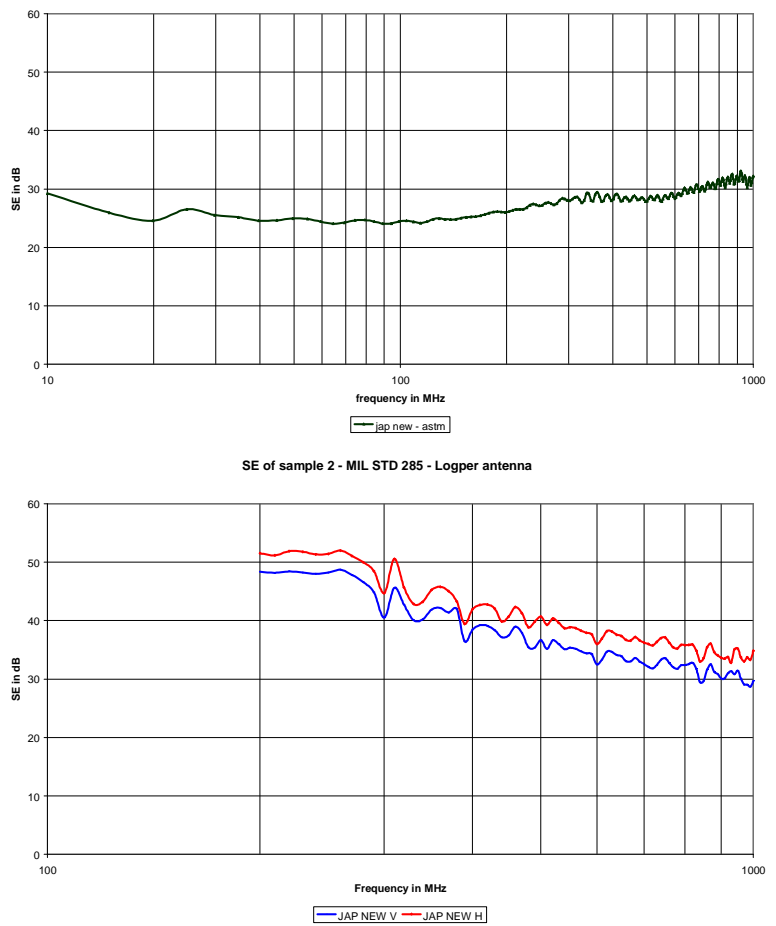

Figure 8. SE of isotropic material, ASTM D4935 (upper) and IEEE 299 logper antenna (lower)
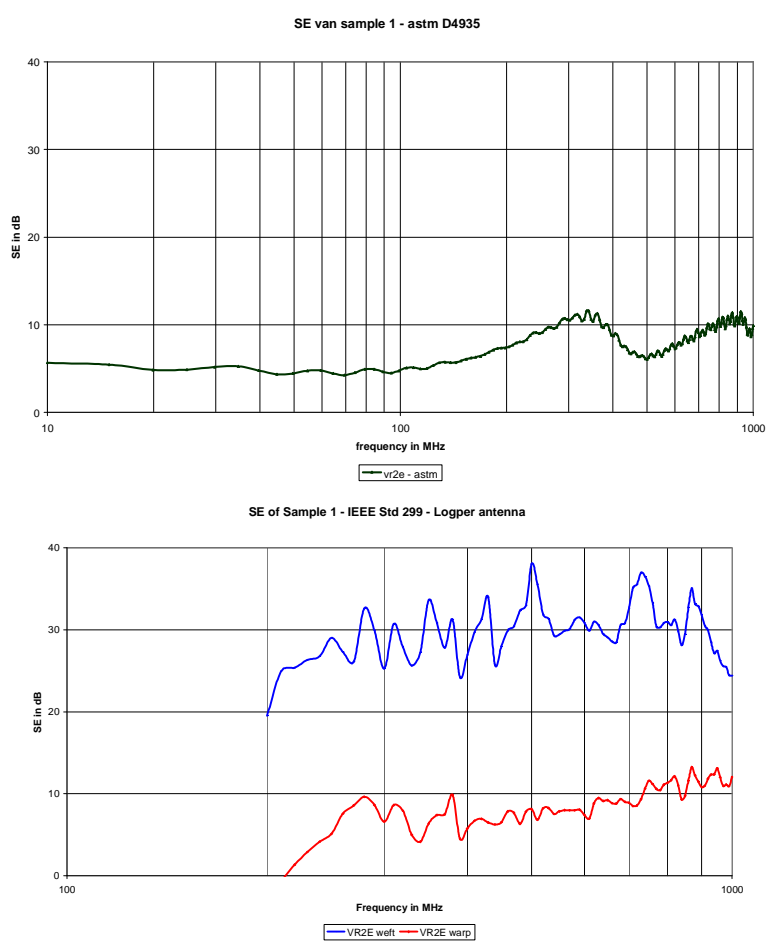

Figure 9. SE of semi-isotropic material, ASTM D4935 (upper) and IEEE 299 logper antenna (lower)
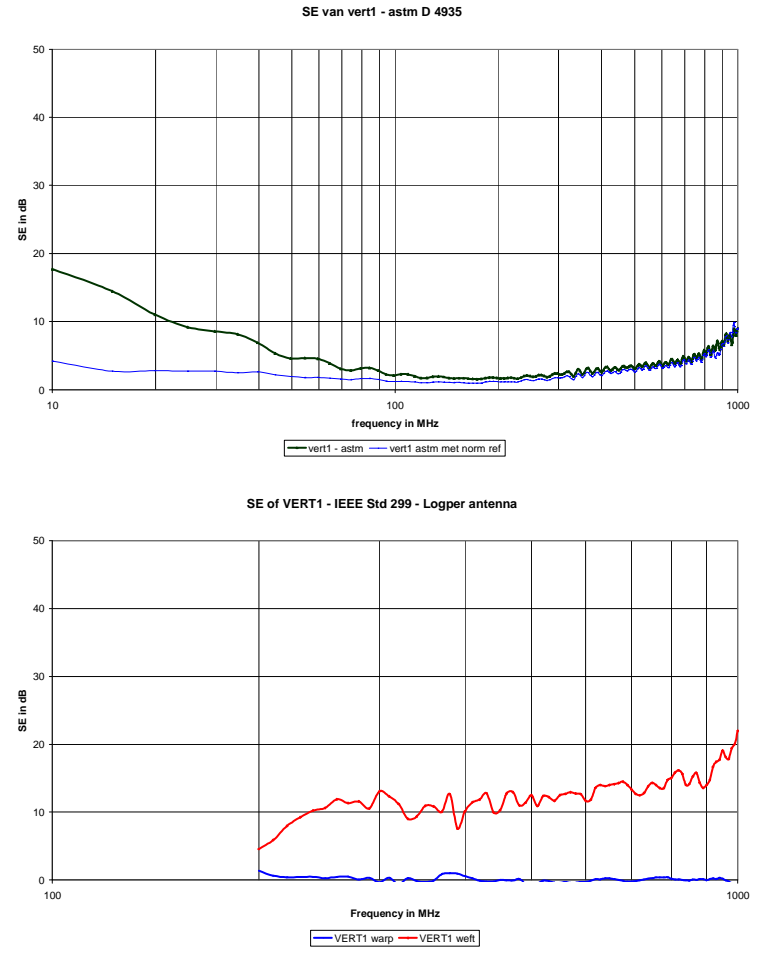

Figure 10. SE of anisotropic material, ASTM D4935 (upper) and IEEE 299 logper antenna (lower)

Due to the size of the window in the IEEE 299 setup, SE measurements are only valuable over $200 \mathrm{MHz}$.

Concerning the isotropic and semi-anisotropic materials, the ASTM D4935 (plane wave) results tend to be lower than the IEEE 229 ones, which still shows a E-field dependent-like SE value.

Another effect that may influence the final SE value measured using IEEE 299, is due to the fact that a conductive wall is interacting in a capacitive way with a dipole at distances shorter than a wavelength. Consequently, the antenna impedance behaves capacitive and is no more matched to the output impedance of the measuring equipment with the samples in place. A lower amplitude of the transmitting wave is generated and a higher SE value is obtained.

Concerning full anisotropic materials, measured SE results using the ASTM D4935 cell tend to the lowest SE values obtained using the IEEE 299 setup. The IEEE 299 setup offers a clear discrimination of directional properties of the material under test. A more detailed analysis is found in reference [5].

It is clear that different SE values are obtained in function of the type of material, even using different standardized measurement methodologies. The question is which SE value must be taken into account in the real environment, with an unknown polarisation of the electromagnetic ambient. 


\section{4. $\mu$ WAVE FREQUENCY RANGE}

In the range above $1 \mathrm{GHz}$, the IEEE 299 setup using horn antenna's is commonly used. But, as an alternative, a setup in free space is also frequently used. But it needs rather large samples of material, in order to avoid "leakage" over the edges of the samples.

As for both measuring setups, horn antenna's are used, a well defined polarization is obtained and directional properties of the materials are observed.

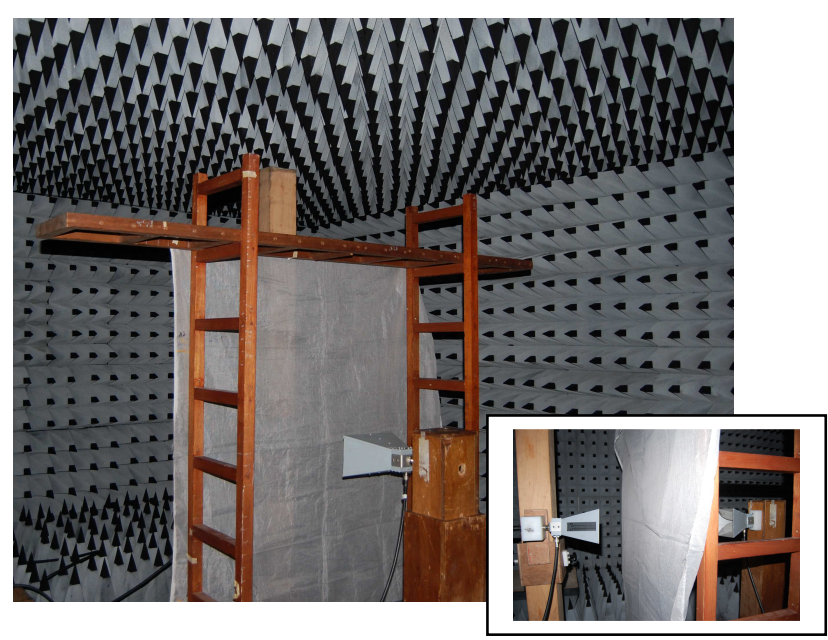

Figure 11. Picture of the free space test setup

The next example shows the results for an isotropic type of material, but with a size of only $1 \times 1 \mathrm{~m}$ in the free space setup.

SE of HVLS1 - IEEE Std 299 - horn antenna
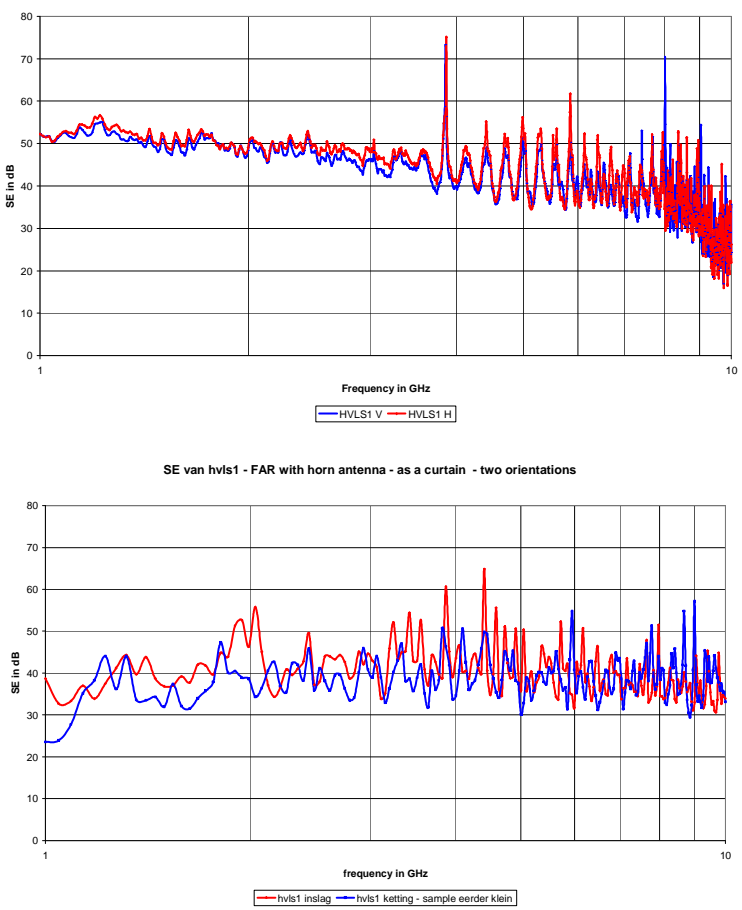

Figure 12. SE of 'small' isotropic material, IEEE 299 horn (upper) and free space horn antenna (lower)
Due to the rather small sample of material, the free space SE tends to be lower than the IEEE 299 SE value, because of some "leakage over the edges".

In case that material samples of larger size are available, no "leakage over the edges" (Fresnel Edge Effect) significantly influencing the measured transmission through/over the material will occur.

The free space setup is a rather simple one and easy to use, but care must given to the fact that no disturbing reflections from walls should occur. A preferred environment to perform these free space shielding measurements is an anechoic room.

The methods will result in a good correlation between both $\mu$ Wave setups, including the discrimination for polarization effects in case of semi-anisotropic and anisotropic materials.

Examples of measured SE are shown in the next figures.
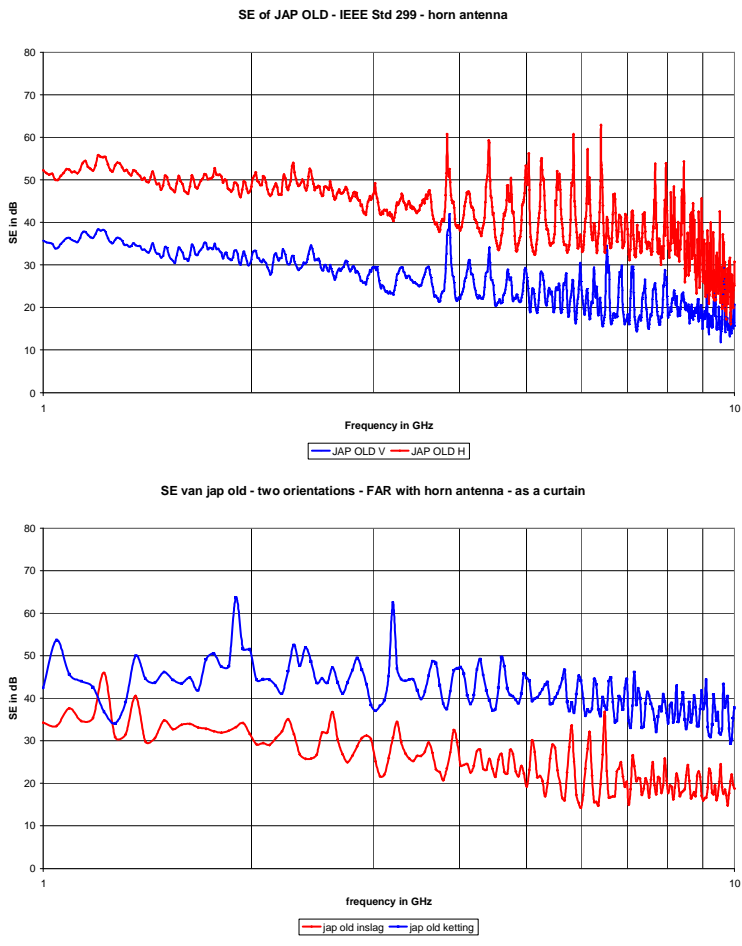

Figure 13. SE of semi-isotropic material, IEEE 299 horn (upper) and free space horn antenna (lower)

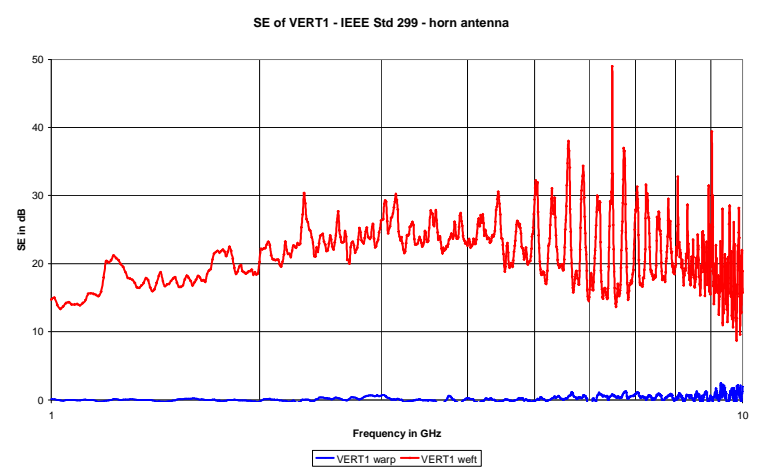




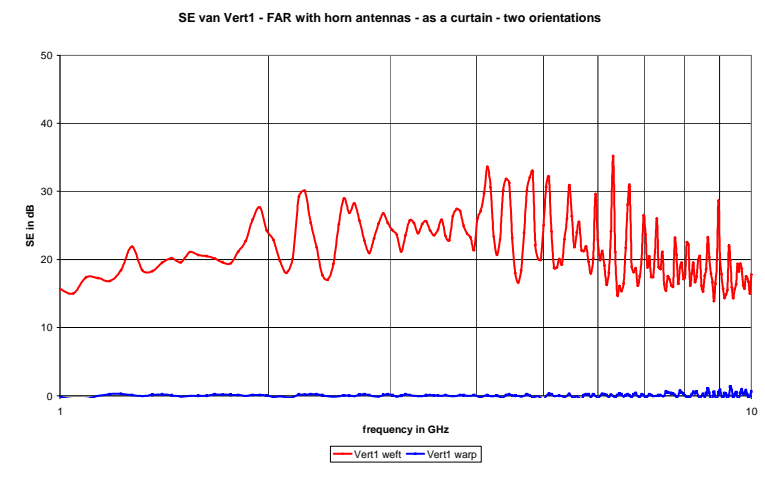

Figure 14. SE of anisotropic material, IEEE 299 horn (upper) and free space horn antenna (lower)

\section{CONCLUSIONS}

In this paper, an overview has been given on the different (semi) standardized measuring methods and methodologies for the characterization of the Shielding Effectiveness of planar composite materials, ranging from isotropic to anisotropic structures.

Although standardized, some differences in SE values have been observed, identified and discussed.

This is especially the case for the IEEE 299 and NSA 94-106 setups at lower frequencies, and for the standardized ASTM D4935 cell and IEEE 299 setup in the $\mathrm{MHz}$ frequency range.

Due to emerging technologies and the expanding frequency range into the high $\mu$ Wave frequencies, new work has to be done. It is referred to a special task force of the IEEE EMC Society, where a PAR on the shielding characterisation of planar materials has recently been approved in order to make a clarification on the different measurement setups in function of the (an)isotropy of the materials, and to expand the frequency range for the SE characterization of planar materials.

\section{REFERENCES}

1. ASTM D4938, "Standard Test Method for Measuring the Electromagnetic Shielding Effectiveness of Planar Materials"

2. IEEE Std. 299 - 2006,"IEEE Standard Method for Measuring the Effectiveness of Electromagnetic Shielding Enclosures", IEEE 2007

3. NSA 94-106, "Specification for shielded enclosures", National Security Agency, Fort George Meade, MD, 1994

4. J. Catrysse et al., "Differences between NSA 94-106 and IEEE 299 LF Magnetic Shielding Measurements", Proc. IEEE International Symposium on EMC, Dresden, 2015

5. J. Catrysse et al., "Shielding Effectiveness of Anisotropic Materials: How to Measure?", Proc. IEEE International Symposium on EMC, Dresden, 2015 
